\title{
DISEÑO DE UN CUESTIONARIO DE SATISFACCIÓN DE ESTUDIANTES PARA UN CURSO DE NIVEL PROFESIONAL BAJO EL MODELO DE APRENDIZAJE INVERTIDO
}

\section{Design of a Student Satisfaction Questionnaire for a Higher Education Course Implementing the Flipped Learning Model}

\author{
MARÍA RAQUEL LANDA CAVAZOS*1 \\ MIGUEL YSRRAEL RAMÍREZ SÁNCHEZ**2 \\ *Instituto Tecnológico de Estudios Superiores \\ de Monterrey. México. Correspondencia: rlanda@itesm.mx \\ **Universidad Internacional Iberoamericana. Correspondencia: \\ miguel.ramirez@unini.edu.mx
}

Recibido: 08-07-2017

Revisado: 14-08-2017

Aceptado: 01-10-2017

\begin{abstract}
Resumen: El presente artículo describe el proceso de diseño y validación de un cuestionario desarrollado ad hoc para evaluar la satisfacción y percepción de los alumnos con respecto al Modelo de Aprendizaje Invertido, aplicado en un curso de Introducción a la Computación de un instituto de educación superior privada en México. El instrumento se sometió a la validación por un panel de expertos, la validación de confiabilidad por medio del coeficiente alfa de Cronbach y la validación de constructo por medio de un análisis factorial exploratorio. Los resultados de este proceso arrojaron un instrumento válido para realizar una evaluación confiable de la satisfacción de los estudiantes en un curso bajo el Modelo de Aprendizaje Invertido.
\end{abstract}

Palabras clave: aprendizaje invertido; aprendizaje activo; innovación pedagógica; análisis factorial; recogida de datos.

\begin{abstract}
This paper describes the process of design and validation of a questionnaire developed ad hoc to evaluate the satisfaction and perception of students with respect to the Flipped Learning Model applied in an Introduction to Computing course in a private university in Mexico. For this purpose, a validation by a panel of experts, the validation of reliability by means of the Chronbach alpha coefficient and the construct validation by means of an exploratory factorial analysis were carried out. The results of the process show that the proposed questionnaire is a valid instrument for a reliable evaluation of student satisfaction in a course implementing the Flipped Learning Model.
\end{abstract}

Keywords: flipped learning; active learning; educational innovation; factor analysis; data collection.

\footnotetext{
1 Ingeniera en Sistemas Electrónicos y magíster en Administración de Tecnologías de Información del Instituto Tecnológico y de Estudios Superiores de Monterrey, Campus Monterrey (México). Profesora de cátedra del Instituto Tecnológico y de Estudios Superiores de Monterrey, Campus Monterrey (México). Actualmente cursa el Doctorado en Educación con enfoque en Tecnología Educativa en el cual centra su investigación en el tema de Aprendizaje Invertido.

${ }^{2}$ Licenciado en Informática, magíster en Dirección Estratégica por la Universidad de Belgrano (Argentina), con doble título en Master in Business Administration por la Universidad de Barcelona (España), con créditos por intercambio académico en la Escola de Administração de Empresas de São Paulo da Fundação Getulio Vargas (Brasil) y doctor en Educación por la Universidad Internacional Iberoamericana (Puerto Rico). Profesor investigador y coordinador en lengua portuguesa del doctorado en proyectos en la Universidad Internacional Iberoamericana (México).
} 


\section{INTRODUCCIÓN}

En la actualidad se encuentran en las aulas universitarias estudiantes denominados millennials, que corresponden a todos aquellos individuos nacidos y criados en la era digital, que poseen características únicas que los diferencian de las generaciones anteriores. Son la primera generación que accede al mundo a través de las nuevas tecnologías desde su alfabetización. Para ellos los dispositivos móviles no fueron un avance científico, sino una realidad, una presencia constante, y en su rol de estudiantes requieren de un nuevo paradigma educativo. Sus características y preferencias, en cuanto a la forma que tienen de aprender, ocasionan que estén en constante conflicto con los métodos de enseñanza tradicionales, ya que no los consideran adecuados a sus necesidades. Para lograr mejores resultados de aprendizaje y de satisfacción en los estudiantes la pedagogía tradicional debe ser modificada considerando estos nuevos requerimientos, entre los que se encuentran: modelos que les permitan un aprendizaje más rápido, la capacidad de hacer más de una tarea a la vez, el ser principalmente visuales, utilizar materiales con hipervínculos, estar conectados por medios electrónicos, aprender haciendo, preferencia por la instrucción divertida y relajada, el obtener gratificación inmediata, incluir la fantasía y juego en el trabajo y finalmente partir del supuesto de que la tecnología es necesaria para su proceso de aprendizaje. Es precisamente aquí donde cobran particular relevancia modelos innovadores de enseñanza como Aprendizaje Invertido.

El Modelo de Aprendizaje Invertido toma el contenido que era expuesto en clase, instrucción dirigida por parte del profesor, y lo reemplaza con lo que tradicionalmente era realizado como tarea, actividades asignadas para completarse por los alumnos en casa, las que se llevan a cabo dentro del aula. Adicionalmente le otorga al alumno la responsabilidad de su propio aprendizaje en un grado mayor, ya que requiere completar el trabajo de preparación previo a la sesión, así como ser más participativo e interactuar con sus compañeros y con el profesor durante el tiempo de clase. Este enfoque trae consigo diversas ventajas que se relacionan con su flexibilidad, el uso de recursos tecnológicos, la capacidad de involucrar a los estudiantes en el proceso de aprendizaje, el liberar tiempo de clase para actividades retadoras y de orden superior. El modelo tiene el potencial de entrenar a los estudiantes en las habilidades requeridas por las empresas del siglo XXI. Es necesario, sin embargo, evaluar a detalle las mejores prácticas y los resultados específicos que se logran con su implementación.

Este modelo se centra en proporcionar a los estudiantes la instrucción inicial a los temas del curso utilizando medios alternativos a la exposición en clase, quienes generalmente hacen uso de las TIC (Tecnologías de Información y Comunicación). Los alumnos tienen la responsabilidad de acceder a los distintos materiales que el profesor 
proporciona antes de la sesión en el aula para conocer y analizar de forma autónoma los conceptos en los que se profundizará por medio de actividades orientadas a la aplicación. Según la Red de Aprendizaje Invertido o Flipped Learning Network (2014):

El aprendizaje invertido es un enfoque pedagógico en el que la instrucción se desplaza de la dimensión del aprendizaje grupal a la dimensión del aprendizaje individual, transformándose el espacio grupal restante en un ambiente de aprendizaje dinámico e interactivo en el que el facilitador guía a los estudiantes en la aplicación de los conceptos y en su involucramiento creativo con el contenido del curso.

Resalta el hecho de que la definición anterior no solo hace referencia a un cambio en el espacio físico donde ocurre la instrucción directa (aula) sino a un nuevo enfoque en la manera en que se lleva a cabo el aprendizaje, mientras el concepto de "aula invertida" implica solamente un cambio de logística en cuanto a cómo y cuándo reciben la información inicial respecto a un tema los alumnos; el "aprendizaje invertido" se enfoca en los procesos de alto nivel cognitivo en los que éstos se involucran. El invertir el aula no traerá consigo de forma directa un proceso de "aprendizaje invertido", para ello es necesario que exista un rediseño completo de las actividades, de tal forma que estas potencien las oportunidades tanto dentro como fuera del aula para lograr un aprendizaje significativo y centrado en el estudiante. La Figura 1 muestra la secuencia de las actividades que propone el Modelo de Aprendizaje Invertido.

Figura 1. Secuencia de actividades realizadas por el alumno en el Modelo de Aprendizaje Invertido

\begin{tabular}{|c|c|c|}
\hline Previo a la sesión & Durante la sesión & Posterior a la sesión \\
\hline $\begin{array}{l}\text { Los estudiantes } \\
\text { acceden a los } \\
\text { materiales } \\
\text { proporcionados por } \\
\text { el profesor para } \\
\text { conocer y analizar } \\
\text { de forma autónoma } \\
\text { los conceptos. }\end{array}$ & $\begin{array}{l}\text { Los estudiantes } \\
\text { profundizan sobre } \\
\text { los conceptos por } \\
\text { medio de } \\
\text { actividades } \\
\text { orientadas a la } \\
\text { aplicación de los } \\
\text { mismos bajo la } \\
\text { supervisión del } \\
\text { profesor. }\end{array}$ & $\begin{array}{l}\text { Se evalúa el } \\
\text { manejo de los } \\
\text { conceptos y los } \\
\text { estudiantes } \\
\text { continúan } \\
\text { profundizando } \\
\text { sobre ellos. }\end{array}$ \\
\hline
\end{tabular}

Fuente: Adaptado de Hamdan, MCKnigth, y Arfstrom (2014) 
De acuerdo con lo presentado por Anderson et al. (2014), en términos de la taxonomía revisada de Bloom, el concepto de "invertir el aprendizaje" implica que los estudiantes están empleando las habilidades cognitivas básicas o de bajo nivel (adquiriendo conocimiento y comprensión) fuera del aula, y enfocándose en las habilidades cognitivas avanzadas como aplicación, análisis, síntesis o evaluación dentro del salón de clases, en donde cuentan con el apoyo de sus pares y del profesor. Este modelo contrasta con el de instrucción tradicional donde el primer contacto con el contenido se realiza mediante la exposición del profesor en el aula y la asimilación de conceptos se basa en las actividades asignadas de tarea, este cambio de paradigma es lo que se llama Modelo de Aprendizaje Invertido. Este enfoque muestra el rol del profesor como un guía dentro del aula en lugar de un expositor de contenido, permitiendo a los estudiantes aprender de forma activa y construir la confianza en sí mismos; proporciona también apoyo y motivación uno a uno a los estudiantes, quienes aprenden de sus compañeros, así como del profesor (Sarawagi, 2013). En la Figura 2 se muestra una comparación entre el Modelo de Aprendizaje Invertido y el Modelo Tradicional bajo la perspectiva de la taxonomía revisada de Bloom.

Figura 2. Modelo de Aprendizaje Invertido comparado con Modelo Tradicional bajo la perspectiva de la Taxonomía revisada de Bloom

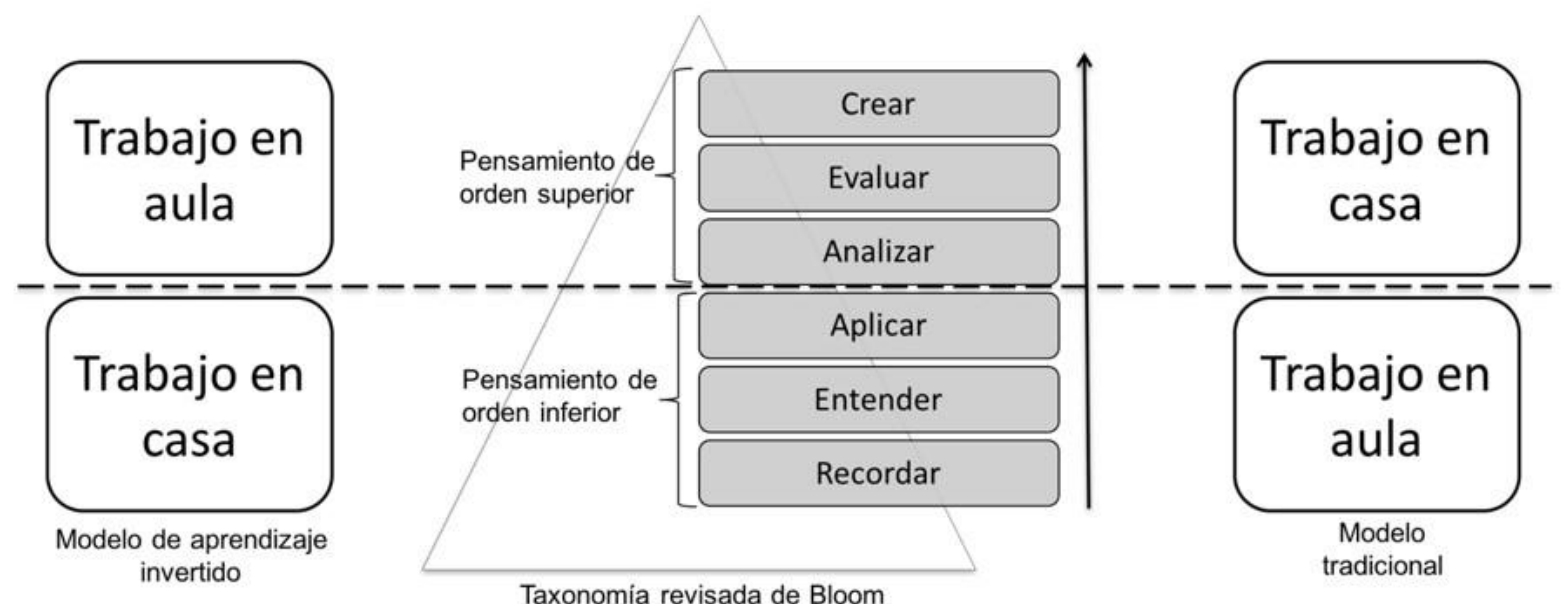

Fuente: Adaptado de Honneycutt y Garrett (2014)

Es importante resaltar que, aunque en la mayoría de las definiciones del Aprendizaje Invertido sobresalen como actores principales en el Modelo de Aprendizaje Invertido el alumno y el profesor, existen otros personajes que participan de forma activa en el modelo, y que además pueden influenciar de forma positiva o negativa los resultados de su implementación. 
De acuerdo con Bergmann y Sams (2014) los padres, los administradores de las instituciones educativas y la tecnología también forman parte de los denominados stakeholders o actores en el Modelo de Aprendizaje Invertido. La Figura 3 muestra de forma gráfica los stakeholders del modelo, es importante entender la función que cada uno de ellos desempeña en su ejecución y desarrollo para lograr potencializar los efectos de la innovación en el aprendizaje y minimizar las posibles barreras de implementación.

Figura 3. Actores del Modelo de Aprendizaje Invertido

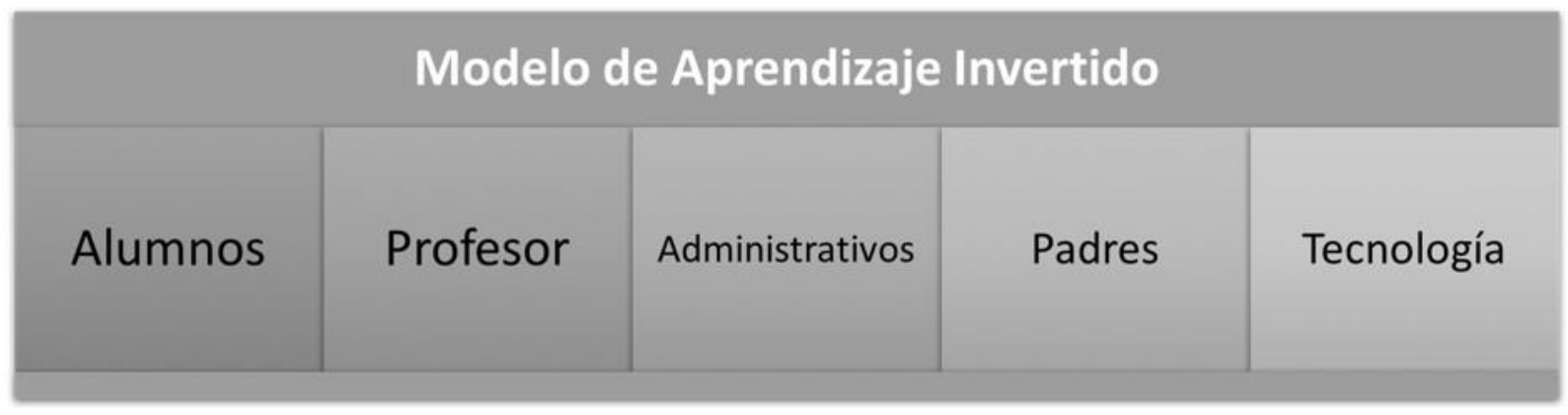

Fuente: Adaptado de Bergmann y Sams (2014)

En primera instancia están los alumnos, ellos son el centro del aprendizaje y deben tener un rol activo para que los objetivos de aprendizaje puedan lograrse de forma exitosa, es importante, sin embargo, explicarles claramente el modelo ya que muy probablemente muchos de ellos no han tenido experiencias previas utilizándolo, por lo que se vuelve indispensable hacer explícitos los comportamientos esperados de ellos tanto dentro como fuera del aula, así como las razones que motivan este cambio de paradigma. De acuerdo a Ozdamli y Asiksoy (2016) el rol de los estudiantes dentro de este modelo se transforma de un receptor pasivo a un promotor activo del conocimiento, esto implica tomar responsabilidad de su propio aprendizaje, ver las exposiciones en video definidas por el profesor antes de la sesión en el aula, así como prepararse revisando los materiales asignados, aprender a su propia velocidad, realizar las interacciones necesarias con su profesor y compañeros, recibiendo y proporcionando retroalimentación, participar en las discusiones dentro del aula, además de participar en las actividades en equipo, entre otras.

Posteriormente, se encuentra el profesor, cuya función es relacionar los procesos de construcción internos del alumno con el conocimiento colectivo culturalmente organizado. Esto implica que el profesor debe orientar y guiar explícita y deliberadamente una actividad mental constructivista. Además de ser el promotor principal del modelo con los demás actores, ya que es quién está propiciando el cambio de modelo en el aula. Para Ozdamli y Asiksoy (2016) el factor más importante en el enfoque del aprendizaje 
invertido es el rol del profesor, que incluye crear las condiciones de aprendizaje basadas en el pensamiento crítico de los estudiantes, modificar el paradigma para en lugar de "transferir" el conocimiento de forma directa, ser una guía y actuar como su facilitador, realizar interacciones uno a uno con los alumnos, aclarar y corregir concepciones erróneas o conceptos entendidos de forma incorrecta por los estudiantes, individualizar el aprendizaje para los alumnos, utilizar las herramientas tecnológicas que mejor se adapten a entorno de aprendizaje, propiciar las condiciones para llevar a cabo discusiones interactivas, incrementar la participación del grupo, compartir los videos de contenido para las actividades fuera del aula y proporcionar retroalimentación basada en estrategias pedagógicas, entre otras.

Siguiendo con los involucrados en este modelo, se encuentran los administrativos; se debe considerar que cualquier cambio de paradigma trae consigo dificultades y resistencia por parte de los alumnos, ya que les demanda salir de su zona de confort, esto impacta aún más al tratarse de un esquema en el que los alumnos serán exigidos como nunca antes, por lo que es de vital importancia que se cuente con el respaldo de la administración, ya sea proporcionando a los profesores los recursos de tiempo, espacio, capacitación o tecnología necesarios para diseñar, desarrollar e implementar las "actividades invertidas", o apoyando con las dudas o inconformidades que puedan surgir en los demás actores.

Por otro lado, y debido a que este modelo implica cambios en la metodología de trabajo tanto dentro como fuera del aula, es muy importante comunicar el cambio de paradigma que se está planeando implementar y explicar claramente los beneficios y resultados esperados a los padres, ya que de otra forma se corre el riesgo de caer en la idea equivocada de que "el profesor no está haciendo su trabajo" y, por ende, entorpecer e incluso impedir que el proceso se desarrolle de forma eficiente. Hay que recordar que los padres de los alumnos fueron educados bajo un esquema tradicional y esto puede sesgar su participación y aceptación del modelo invertido si no se aclaran sus conceptos.

Finalmente, y como último actor en el modelo, se encuentra la tecnología, este elemento es parte fundamental, ya que se asocia de forma directa con la pedagogía centrada en el alumno y representa un medio para proveer al estudiante de un ambiente de aprendizaje enriquecedor y efectivo, además de ser un facilitador esencial que posibilita la flexibilidad propia del modelo. Aunque no se define como un requisito indispensable, se considera que puede dar un valor agregado sustancial para integrar las experiencias de aprendizaje fuera y dentro del aula de forma eficiente.

De acuerdo a Gillispie (2016) una implementación exitosa del Modelo de Aprendizaje Invertido debe tener tres objetivos: permitir a los estudiantes convertirse en pensadores críticos, involucrar de forma completa y profunda tanto a los estudiantes como a los profesores en el proceso de aprendizaje y estimular el desarrollo de un entendimiento profundo y significativo del contenido. 
Como se menciona anteriormente, los estudiantes en conjunto con el docente son los principales involucrados en el Modelo de Aprendizaje Invertido, por lo que es importante comprender las características que dichos alumnos aportan al proceso de aprendizaje. De acuerdo con Prensky (2001) los millennials son todos aquellos individuos que nacieron entre 1982 y 2002. La literatura hace referencia a ellos como la generación I, la generación de red, nexters o los nativos digitales. Sin importar el término que se utilice para nombrarlos, lo que los caracteriza es que nacieron en la era de la información, por lo que acceden y utilizan la tecnología de forma innata y en un grado mucho mayor que las generaciones que los preceden. Debido a que nacieron y crecieron en la era digital, la forma en que piensan y procesan la información es significativamente distinta del resto de las generaciones anteriores, esto impacta de forma directa en su estilo de aprendizaje y sus preferencias hacia los métodos de enseñanza. Actualmente una gran mayoría de ellos se encuentran en las universidades preparándose para integrarse a la vida productiva en el corto plazo.

Según McMahon y Pospisil (2005), un proceso de enseñanza-aprendizaje exitoso requiere del entendimiento y de una apreciación de las necesidades de los estudiantes, sus antecedentes, intereses y estilos de aprendizaje. En este sentido se han identificado diversas características que definen a los millennials, entre las que se destacan: una preferencia por estar conectados a la red 24/7, tendencia a desempeñarse en ambientes multitarea, preferencia por las actividades en equipo, además de que aprecian el componente social del proceso de aprendizaje.

Por otra parte, como resultado de sus características particulares, que permean sus preferencias hacia el proceso de aprendizaje, los millennials se sienten fuera de lugar en las aulas tradicionales. El método tradicional enfocado en la exposición del profesor que fue muy popular en la era industrial no parece ser el adecuado para estos alumnos que nacieron y crecieron en la era de la información; ellos quieren ser valorados, respetados y escuchados por las instituciones educativas; requieren experiencias de aprendizaje colaborativo y retroalimentación rápida, además de tener muy poca tolerancia hacia el aburrimiento. Más que nunca, los docentes están siendo puestos a prueba por el reto que estos estudiantes representan, el mantenerlos atentos, enfocados y motivados en el proceso de aprendizaje requiere de un profundo entendimiento de sus características y necesidades. El profesor del siglo XXI debe entender a los millennials, sus estudiantes, quiénes son, cómo aprenden y qué necesitan, para poder ofrecerles experiencias de aprendizaje significativas; el rol del profesor necesita ir más allá de un experto en contenido de tal forma que pueda lograr que los estudiantes, quienes finalmente se convertirán en profesionales, sean capaces de auto administrar su proceso de aprendizaje. Diversas investigaciones en psicología cognoscitiva han revelado que los estudiantes alcanzan un mayor grado de procesamiento de la información por medio de la metacognición y el aprendizaje activo, estableciendo incluso que este tipo de aprendizajes supera en muchos sentidos a los métodos expositivos. Incluso se sugiere 
que los métodos basados en el aprendizaje activo incrementan el desempeño, la motivación y la capacidad de retención de los estudiantes (D'Souza y Rodrígues, 2015).

El Modelo de Aprendizaje Invertido tiene componentes que pueden ser atractivos para todas las generaciones involucradas en el ambiente académico, para los baby boomers que prefieren el ambiente tradicional, la instrucción didáctica, aunque ocurre fuera del aula, puede llevarse a cabo inicialmente de forma tradicional si se prefiere. Los estudiantes de la generación X, por su parte, disfrutan aprendiendo de videos que utilizan alta tecnología y aprendizaje asistido por computadora. Los estudiantes de la generación Y son altamente colaborativos, eficientes con la tecnología y se benefician de la solución de problemas en grupo. En el aprendizaje invertido, el contenido es entregado a los estudiantes en formatos que atraen a la generación $X$, mientras que la solución de problemas de forma grupal es el componente que engancha a la generación $Y$ (Gillispie, 2016).

En la literatura existe evidencia indirecta de que el Modelo de Aprendizaje Invertido puede incrementar tanto la satisfacción de los estudiantes como sus calificaciones, alineándose a sus características y sus requerimientos personales, sin embargo los resultados positivos que los docentes han obtenido alrededor del mundo han sido documentados principalmente a través de medios no formales como blogs y sitios web personales, estos resultados han incrementado el interés de la comunidad científica por el tema como se observa en Bailey (2015); Bergmann (2012);Bishop y Verleger (2013); Fautch (2015); Flumerfelt y Green (2013); Hao (2016); Herreid y Schiller (2013); Joanne y Lateef (2014); González-Gómez, Jeong, Airado y Cañada-Cañada (2016); McLean, Attardi, Faden y Goldszmidt (2016); Murphy, Chang y Suaray (2015); Ojennus (2015); Sams y Aglio (2017); Thai, De Wever y Valcke (2017) y Tucker (2012).

Adicionalmente para Johnson, Adams, Estrada y Freeman (2014) el Modelo de Aprendizaje Invertido forma parte de un movimiento pedagógico mayor que involucra innovaciones, las que conjuntan el aprendizaje híbrido, el aprendizaje basado en preguntas y otros enfoques instruccionales y herramientas que tienen por objetivo propiciar un aprendizaje flexible, activo y más centrado en el estudiante. Siete años después de su primera implementación reconocida en 2007, existe una cantidad considerable, que cada vez se incrementa más, de implementaciones exitosas de este modelo, por lo que se consideró como una de las seis tendencias en Educación Superior.

En una versión posterior en Johnson et al. (2015) se sigue identificando el Aprendizaje Invertido como una de las seis tendencias en Educación Superior que serán adoptadas en un año o menos por las universidades y que se espera tengan un impacto global en el aprendizaje, la enseñanza y la investigación educativa. De acuerdo a las estadísticas que muestran en los Estados Unidos de América el 29\% de los profesores universitarios se encuentran aplicando Aprendizaje Invertido, mientras que un $27 \%$ planean hacerlo en el siguiente año. Ejemplos de estas implementaciones se encuentran en la Universidad Swinburne, la Universidad de British Columbia, la Universidad de South 
Australia, la Universidad de Boston, la Universidad Canadiense y la Universidad de Ohio State, entre otras.

Por su parte, De Araujo, Otten y Birisci (2017) refieren que a pesar de que las investigaciones formales respecto al Modelo de Aprendizaje Invertido aún son escasas, las implementaciones de este modelo instruccional no lo son; examinando las perspectivas y experiencias de los docentes que han optado por implementar en sus aulas esta innovación es posible identificar formas de soportar el modelo y reflexionar respecto a si las motivaciones para llevar al aula esta metodología se alinean con las expectativas de las instituciones educativas y los beneficios potenciales que se esperan, entre los que sobresale la satisfacción de los estudiantes, que engloba conceptos como la motivación y disposición de los alumnos hacia este nuevo modelo.

En general, las investigaciones respecto a la eficacia del Modelo de Aprendizaje Invertido en la educación superior se han centrado, en primera instancia, en el impacto en las evaluaciones numéricas (calificaciones) y, de forma más reciente y en menor grado, en las percepciones de los estudiantes hacia este nuevo paradigma. Las percepciones de los estudiantes proporcionan una oportunidad única de analizar e identificar mejores prácticas que permitan lograr de forma exitosa los objetivos de aprendizaje, lo anterior bajo el precepto de que en la medida de que los estudiantes se involucren, interesen y comprometan con el proceso se lo apropiarán. El entender los factores claves que impactan y se relacionan con la satisfacción de los estudiantes en las experiencias de aprendizaje invertido es indispensable para lograr implementaciones exitosas.

Es precisamente el análisis de la satisfacción de los estudiantes bajo el Modelo de Aprendizaje Invertido lo que pretende ser valorado por medio del instrumento denominado "Cuestionario de satisfacción y percepción de alumnos acerca del impacto de las TIC en cursos que implementan actividades bajo el Modelo de Aprendizaje Invertido". El proceso de diseño y su validación se detallan en los siguientes apartados.

\section{DISEÑO DEL INSTRUMENTO}

El instrumento diseñado consta de 23 preguntas segmentadas en tres secciones: 1) datos demográficos, 2) datos cuantitativos respecto a la percepción de los alumnos y 3) datos cualitativos respecto a la percepción de los alumnos. El análisis que aquí se presenta está centrado en la segunda sección del cuestionario, que se compone de 16 preguntas que consideran elementos cuantitativos usando una escala Likert de 5 niveles, en donde los valores van de 1 a 5 , siendo 1 el valor correspondiente a la respuesta con menor valor y 5 la respuesta con mayor valor. El análisis estadístico realizado se centra en preguntas cerradas de opción múltiple. 
De acuerdo con Franklin y Walker (2010) existen numerosas ventajas de utilizar preguntas cerradas en un instrumento de recolección de datos, siendo la más importante el hecho de que no sobrecargan a los respondientes, así como el que la captura de la información es más simple y eficiente. Este tipo de preguntas son más sencillas y rápidas de contestar, ya que solo implican la selección de las opciones planteadas en el instrumento, por lo que no se requiere que quien responde formule una respuesta y la exprese en sus propias palabras, por lo que es más probable que el cuestionario logre recabar la información deseada. Adicionalmente, los datos generados son más fáciles de analizar debido a que las respuestas son más consistentes y se encuentran agrupadas.

Por su parte, la implementación del instrumento de recolección de datos se realizó por medio de la herramienta Google Forms, que permite aplicar encuestas a través de Internet. De acuerdo a De Leeuw, Dillman y Hox (2011) la recolección de datos a través de formatos en Internet tiene diversas ventajas como: ser menos intrusivas y más privadas que otros tipos de medios, permitir aplicar grandes cantidades de cuestionarios en un tiempo muy corto y con un costo considerablemente bajo y, en caso de ser necesario, llegar a poblaciones en distintas ubicaciones geográficas sin necesidad de trasladarse.

En lo que corresponde al diseño del cuestionario se consideró lo expuesto en De Leeuw, Dillman y Hox (2011) donde se establece que existen 3 etapas por las que deben pasar los instrumentos de recolección de datos para poder ser utilizados: la etapa de desarrollo, la etapa de evaluación de preguntas y la etapa de ensayo final. La etapa de desarrollo corresponde al tiempo designado para el trabajo de preparación e investigación previa que se requiere realizar antes de escribir alguna de las preguntas del instrumento en cuestión, es un período en el que se analiza de forma detallada y a profundidad la literatura existente respecto al tema en cuestión e incluso se consultan expertos, en caso de considerarlo necesario y relevante para el diseño del cuestionario.

En la segunda etapa, la de evaluación, se realiza una prueba del cuestionario en una población piloto, el objetivo de esta etapa es asegurar que cada una de las preguntas que componen el instrumento cumple con los principios de un buen diseño. Finalmente, en la tercera etapa se realiza el ensayo final, en donde el objetivo es someter el cuestionario completo a prueba, lo anterior bajo las mismas condiciones (o lo más similares posible) en las que será utilizado al realizar la recolección de datos de la investigación, esto utilizando una muestra mucho más grande que la de la etapa anterior. A menos que la investigación explícitamente lo requiera, en muchos casos esta última etapa no es indispensable y es más recomendable realizar de nuevo la etapa anterior para validar una vez más el instrumento con los cambios que se hayan realizado.

El diseño de cuestionarios es un proceso iterativo que fluctúa entre el desarrollo de las preguntas y su evaluación por medio de pruebas, los cambios son parte integral de este proceso y se realizan de forma continua, las preguntas propuestas son 
esbozadas y evaluadas, revisadas y modificadas hasta que una versión final del cuestionario es generada; la etapa final de este proceso llega cuando se decide que no son necesarios más cambios, entonces se procede a la impresión o programación del instrumento según sea el caso (Franklin y Walker, 2010).

\section{METODOLOGÍA}

Para establecer la validez del instrumento en cuestión, éste se sometió a la validación por un panel de expertos, la validación de confiabilidad por medio del coeficiente alfa de Cronbach y la validación de constructo por medio de un análisis factorial exploratorio.

De acuerdo a Franklin y Walker (2010) la revisión de cuestionarios por un panel de expertos tiene como ventajas que es rápida, de un costo moderado, puede cubrir una amplia variedad de problemas potenciales que van desde errores de ortografía, gramática 0 de redacción hasta problemas con los conceptos que se están operacionalizando en el cuestionario, adicionalmente cubre aspectos cognitivos de los respondientes, puede develar dificultades potenciales para el entrevistador (en caso de que éste exista) y puede revelar posibles problemas para el análisis de datos.

Por su parte, el método de consistencia interna basado en el alfa de Cronbach permite estimar la fiabilidad de un instrumento de medida a través de un conjunto de ítems que se espera que midan el mismo constructo o dimensión teórica. Para calcular este coeficiente solamente es necesaria una aplicación del instrumento de medición, los resultados que arroja son valores entre 0 y 1 . Su principal ventaja es que los elementos no requieren dividirse en dos mitades, solamente se aplican y se calcula el coeficiente (Hernández et al., 2014).

El valor del coeficiente muestra la consistencia interna, es decir, la correlación existente entre cada una de las preguntas del instrumento; un resultado por encima a 0.7 muestra una fuerte correlación entre las preguntas, mientras que un valor por debajo de este número establece una relación débil entre ellas, en cuyo caso se debe rediseñar el instrumento. El valor mínimo que se considera aceptable para el coeficiente alfa de Cronbach es 0.7 , cualquier valor por debajo este número refleja una consistencia interna baja (Oviedo y Campo, 2005).

En lo que respecta a la evaluación de la validez de constructo para Pérez-Gil, Chacón y Moreno (2000) entre las técnicas estadísticas más utilizadas para este fin destaca el análisis factorial, que agrupa una serie de procedimientos de análisis multivariable que determinan la relación entre cada una de las variables, además permite estudiar la interdependencia existente entre ellas, así como establecer índices con variables que miden conceptos similares. 
Comprende el estudio estadístico de distintas variables medidas en elementos de una población con los siguientes objetivos: 1) resumir los datos mediante un pequeño conjunto de nuevas variables, construidas como transformaciones de las originales, con la mínima pérdida de información; 2) encontrar grupos en los datos con propiedades similares; 3) clasificar nuevas observaciones en grupos definidos y 4) relacionar dos conjuntos de variables.

De acuerdo a Martínez y Sepúlveda (2012) el análisis factorial exploratorio (AFE) tiene dos objetivos principales, de inicio pretende desde un enfoque de exploración esbozar una nueva estructura interna, generando grupos de variables o factores a partir de las variables originales, en este tipo de análisis se asume que el investigador no cuenta con información previa respecto a la estructura que se va a generar o que ésta es mínima.

La segunda motivación para realizar un AFE es reducir la cantidad de variables identificando aquellas que tengan poca relevancia o un alto grado de colinealidad. En el caso particular de la investigación el objetivo principal de realizar un análisis factorial fue la validación del instrumento de recolección de datos, esto, al generar nuevos factores o variables latentes que agrupen los reactivos existentes, además de identificar posibles ítems redundantes; ambas cuestiones se consideran como parte del enfoque de un AFE. Para determinar si es viable realizar un AFE es necesario identificar si existe correlación entre las variables, esto es posible inspeccionando la matriz de correlaciones y determinando si los valores que se muestran son superiores a 0.30 para todos los casos, ya que en caso contrario no se recomienda aplicar el AFE.

De forma específica los pasos que se realizaron para confirmar la validez son los siguientes: 1) se elaboró el instrumento de evaluación denominado "Cuestionario de satisfacción y percepción de alumnos en cursos que implementan actividades bajo el Modelo de Aprendizaje Invertido" para medir la satisfacción de los alumnos, 2) se realizó una revisión cualitativa del instrumento por medio de un panel de expertos integrado por los profesores que imparten la asignatura de Introducción a la Computación en la institución educativa en la que se implementó el Modelo de Aprendizaje Invertido, 3) se realizó un primer piloto para efectuar la validación de confiabilidad del instrumento por medio del índice alfa de Cronbach y 4) se realizó un segundo piloto para efectuar una validación de constructo por medio de un análisis factorial exploratorio del instrumento. Finalmente, y después de analizar los resultados del análisis factorial se procedió a la aceptación del cuestionario. La Figura 4 muestra de forma gráfica el proceso que se siguió para la validación del instrumento. 
Figura 4. Fases del proceso de validación

Diseño del instrumento "Cuestionario de satisfacción y percepción de alumnos acerca del impacto de las TIC en cursos que implementan actividades bajo el Modelo de Aprendizaje Invertido"

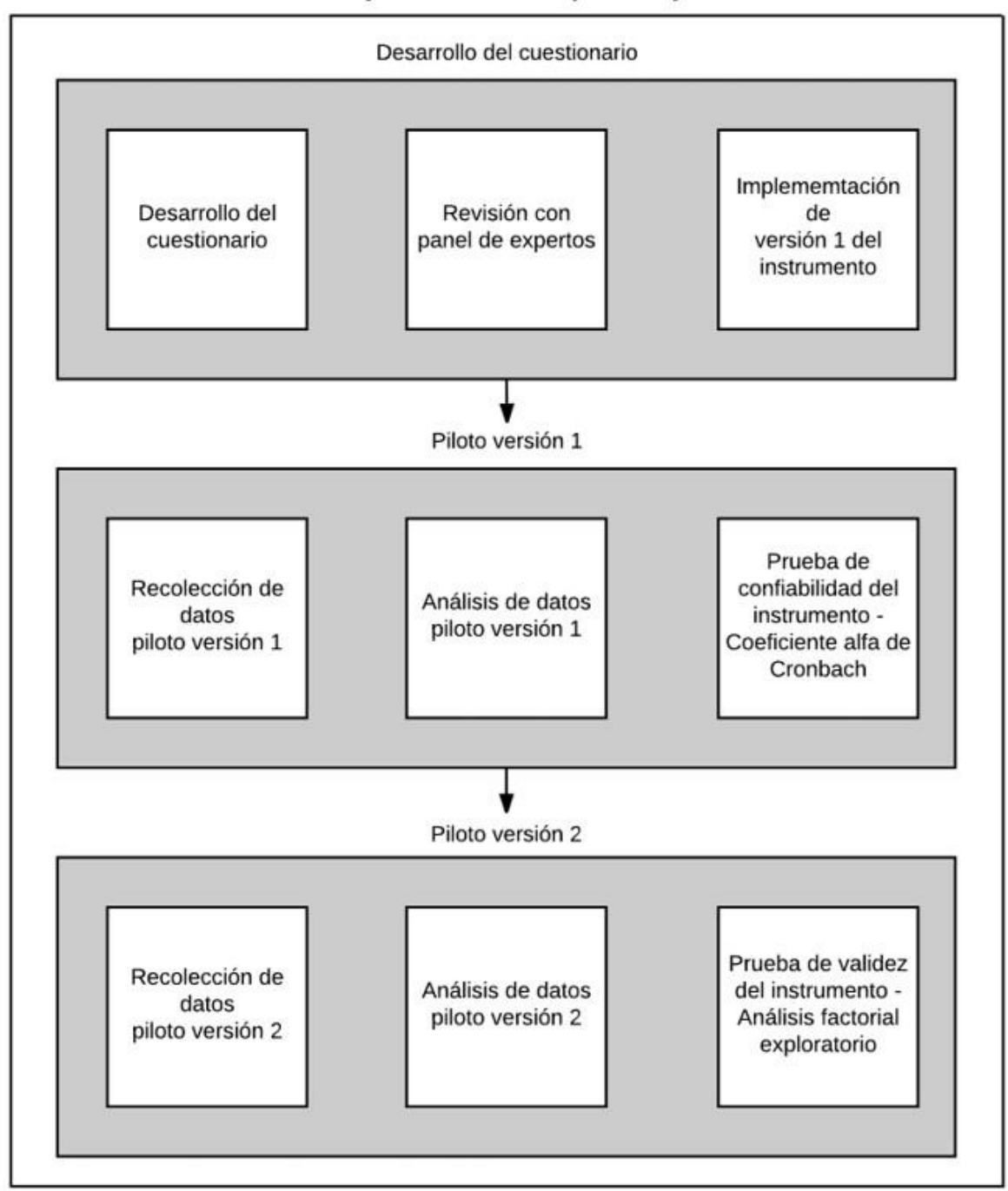

Fuente: Elaboración propia (2017)

\section{ANÁLISIS DE RESULTADOS}

Para realizar el análisis de los resultados obtenidos del proceso de validación cuantitativa del instrumento, el tratamiento de los datos se realizó por medio del software Minitab (http://www.minitab.com), diseñado para realizar análisis estadísticos básicos y avanzados. Se realizaron las pruebas de confiabilidad y de validez de constructo utilizando las funcionalidades propias de este programa. 


\section{Validación cualitativa del instrumento}

Previo a la realización del piloto versión 1 , al inicio del período académico de enero a mayo 2016, se sometió a revisión de todos los profesores que componen la cátedra de la asignatura de Introducción a la Computación de la institución el "Cuestionario de satisfacción y percepción de alumnos en cursos que implementan actividades bajo el Modelo de Aprendizaje Invertido", con el fin de, a través de una revisión de expertos, establecer si las preguntas eran las indicadas para evaluar la satisfacción de los estudiantes del curso. El panel estuvo integrado por la coordinadora de la materia y 5 profesores que habían impartido la asignatura previamente al menos una vez.

Los resultados de esta evaluación de expertos fueron satisfactorios, el panel validó las preguntas incluidas en el cuestionario, no se realizaron correcciones de puntuación y redacción para mejorar la sintaxis de las preguntas. Los cambios solicitados al instrumento fueron los siguientes:

1) Agregar preguntas para recabar datos demográficos correspondientes al semestre y carrera de los estudiantes.

2) Agregar un reactivo con el fin de identificar si los estudiantes han estado expuestos a alguna implementación del Modelo de Aprendizaje Invertido previamente.

Posterior a esta validación por el panel de expertos se realizó la implementación del cuestionario en la herramienta de Google Forms, de tal forma que estuviera disponible para ser utilizado como instrumento de recolección de datos en las etapas subsecuentes.

\section{Validación cuantitativa del instrumento}

Con el fin de validar la confiabilidad del instrumento se realizó un primer piloto donde el cuestionario se aplicó a 36 alumnos de la asignatura de Introducción a la Computación durante el semestre de enero a mayo de 2016. Para realizar la validación de confiabilidad se analizaron los datos obtenidos con el fin de determinar el coeficiente alfa de Cronbach. Como se menciona anteriormente para esta validación se analizaron específicamente los reactivos del 2 al 17, que corresponden a preguntas cerradas que utilizan una escala Likert, en la Tabla 1 se muestra el detalle de las preguntas. 
Tabla 1. Preguntas utilizadas en el análisis de validez interna

\begin{tabular}{ll}
\hline Preguntas & Escala
\end{tabular}

2. En general, ¿qué tan confiado te sentiste respecto al material de la sesión después de

1 a 5 haber visto el video?

3. En general, ¿qué tan confiado te sentiste respecto al material de la sesión después de $\quad 1$ a 5 haber visto el video y haber realizado los problemas de clase?

4. Al día de hoy considero que me siento confiado(a) al resolver un problema de la clase $\quad 1$ a 5 utilizando el Modelo de Aprendizaje Invertido.

5. Basado en tu experiencia en esta clase, ¿qué tan valioso consideras el material en 1 a 5 video para tu aprendizaje?

6. Considero que el Modelo de Aprendizaje Invertido me ofrece más oportunidad de 1 a 5 conocer y colaborar con mis compañeros que el Modelo Tradicional.

7. Me siento más motivado a realizar las actividades del curso en el Modelo de 1 a 5 Aprendizaje Invertido.

8. Me siento más motivado a participar en clase usando el Modelo de Aprendizaje 1 a 5 Invertido.

9. Considero que el Modelo de Aprendizaje Invertido favorece más la comunicación entre 1 a 5 el profesor y los alumnos que el Modelo Tradicional.

10. Considero que el Modelo de Aprendizaje Invertido favorece más la comunicación con 1 a 5 mis compañeros que el Modelo Tradicional.

11. Con el Modelo de Aprendizaje Invertido me es más fácil externar mis dudas y opiniones $\quad 1$ a 5 en el aula.

12. Puedo decir que el Modelo de Aprendizaje Invertido hace que el contenido del curso 1 a 5 sea más fácil de entender en comparación con una clase "tradicional".

13. Puedo decir que el Modelo de Aprendizaje Invertido hace que el contenido del curso $\quad 1$ a 5 sea más fácil de entender en comparación con una clase "tradicional".

14. Considero que el Modelo de Aprendizaje Invertido me ayuda a desarrollar habilidades $\quad 1$ a 5 que serán de valor en mi desarrollo profesional.

15. En el futuro me gustaría cursar otras materias bajo el Modelo de Aprendizaje Invertido. $\quad 1$ a 5

16. Recomendaría a otros estudiantes cursar materia(s) bajo el Modelo de Aprendizaje $\quad 1$ a 5 Invertido.

17. Después de esta experiencia, considero que he dominado el Modelo de Aprendizaje 1 a 5 Invertido.

18. En general me agradó trabajar durante el curso con el Modelo de Aprendizaje Invertido. $\quad 1$ a 5

NOTA. Todos los reactivos utilizan una escala Likert de 5 niveles, en donde 1 corresponde al valor más bajo y 5 al más alto.

Fuente: Elaboración propia (2017) 
El valor obtenido para el coeficiente alfa de Cronbach fue de 0.9699 , lo que indica una alta consistencia y validez interna. La Figura 5 muestra los resultados del tratamiento de los datos arrojados por el programa Minitab 17.

Figura 5. Análisis de confiabilidad del instrumento arrojado por el programa Minitab

Welcome to Minitab, press Fl for help.

Item Analysis of Q2, Q3, Q4, Q5, Q6, Q7, Q8, Q9, Q10, Q11, Q12, Q13, Q14, Q15, Q16, Q17

Correlation Matrix

\begin{tabular}{|c|c|c|c|c|c|c|c|c|c|c|c|c|c|c|c|}
\hline 03 & $\begin{array}{c}02 \\
0.746\end{array}$ & Q3 & Q4 & Q5 & 06 & Q7 & $Q 8$ & 09 & 010 & Q11 & Q12 & 013 & Q14 & Q15 & Q16 \\
\hline$Q 4$ & 0.732 & 0.716 & & & & & & & & & & & & & \\
\hline 25 & 0.777 & 0.797 & 0.676 & & & & & & & & & & & & \\
\hline 26 & 0.605 & 0.481 & 0.605 & 0.403 & & & & & & & & & & & \\
\hline 27 & 0.651 & 0.659 & 0.675 & 0.626 & 0.713 & & & & & & & & & & \\
\hline 08 & 0.562 & 0.643 & 0.562 & 0.554 & 0.640 & 0.857 & & & & & & & & & \\
\hline Q9 & 0.510 & 0.495 & 0.341 & 0.450 & 0.685 & 0.744 & 0.813 & & & & & & & & \\
\hline Q10 & 0.376 & 0.449 & 0.462 & 0.292 & 0.751 & 0.749 & 0.730 & 0.769 & & & & & & & \\
\hline Q11 & 0.543 & 0.741 & 0.626 & 0.537 & 0.498 & 0.642 & 0.696 & 0.588 & 0.546 & & & & & & \\
\hline 012 & 0.779 & 0.786 & 0.653 & 0.795 & 0.575 & 0.723 & 0.690 & 0.573 & 0.508 & 0.747 & & & & & \\
\hline 013 & 0.736 & 0.726 & 0.736 & 0.736 & 0.746 & 0.777 & 0.772 & 0.635 & 0.561 & 0.680 & 0.766 & & & & \\
\hline 014 & 0.686 & 0.646 & 0.619 & 0.522 & 0.760 & 0.789 & 0.811 & 0.588 & 0.637 & 0.601 & 0.745 & 0.804 & & & \\
\hline 015 & 0.755 & 0.748 & 0.708 & 0.729 & 0.698 & 0.842 & 0.865 & 0.682 & 0.616 & 0.702 & 0.843 & 0.880 & 0.894 & & \\
\hline Q16 & 0.809 & 0.859 & 0.831 & 0.765 & 0.668 & 0.692 & 0.625 & 0.458 & 0.492 & 0.618 & 0.744 & 0.825 & 0.714 & 0.783 & \\
\hline 017 & 0.734 & 0.762 & 0.734 & 0.750 & 0.630 & 0.803 & 0.842 & 0.621 & 0.605 & 0.679 & 0.761 & 0.851 & 0.809 & 0.887 & 0.790 \\
\hline
\end{tabular}

Cel1 Contents: Pearson correlation

Cronbach's alpha $=0.9699$

Elaboración propia (2017)

Posterior al análisis de consistencia y validez interna con el coeficiente alfa de Cronbach se realizó un segundo piloto durante el período de verano de 2016, donde se aplicó el instrumento a 36 alumnos de la asignatura de Introducción a la Computación. La Figura 6 muestra la matriz de correlaciones generada por el software Minitab 17 para cada uno de los ítems que forman parte del análisis. 
Figura 6. Matriz de correlaciones de variables del segundo piloto arrojado por el programa Minitab

Item Analysis of Q2, Q3, Q4, Q5, Q6, Q7, Q8, Q9, Q10, Q11, Q12, Q13, Q14, Q15, Q16, Q17

\begin{tabular}{|c|c|c|c|c|c|c|c|c|c|c|c|c|c|c|c|}
\hline & Q2 & 03 & Q4 & Q5 & Q6 & Q7 & Q8 & 09 & Q10 & Q11 & 012 & Q13 & Q14 & Q15 & Q16 \\
\hline Q3 & 0.591 & & & & & & & & & & & & & & \\
\hline 04 & 0.689 & 0.571 & & & & & & & & & & & & & \\
\hline 25 & 0.550 & 0.588 & 0.405 & & & & & & & & & & & & \\
\hline Q6 & 0.581 & 0.400 & 0.622 & 0.427 & & & & & & & & & & & \\
\hline 27 & 0.547 & 0.512 & 0.641 & 0.454 & 0.706 & & & & & & & & & & \\
\hline 08 & 0.472 & 0.375 & 0.644 & 0.468 & 0.644 & 0.790 & & & & & & & & & \\
\hline 09 & 0.488 & 0.497 & 0.633 & 0.444 & 0.595 & 0.653 & 0.577 & & & & & & & & \\
\hline Q10 & 0.550 & 0.415 & 0.709 & 0.414 & 0.734 & 0.595 & 0.626 & 0.723 & & & & & & & \\
\hline Q11 & 0.495 & 0.255 & 0.534 & 0.401 & 0.478 & 0.621 & 0.698 & 0.590 & 0.559 & & & & & & \\
\hline 012 & 0.536 & 0.365 & 0.745 & 0.409 & 0.649 & 0.715 & 0.819 & 0.545 & 0.596 & 0.545 & & & & & \\
\hline 013 & 0.487 & 0.406 & 0.587 & 0.472 & 0.683 & 0.606 & 0.678 & 0.514 & 0.714 & 0.431 & 0.788 & & & & \\
\hline Q14 & 0.590 & 0.547 & 0.716 & 0.613 & 0.732 & 0.799 & 0.788 & 0.692 & 0.690 & 0.582 & 0.886 & 0.797 & & & \\
\hline 215 & 0.601 & 0.566 & 0.738 & 0.621 & 0.704 & 0.764 & 0.755 & 0.635 & 0.653 & 0.546 & 0.840 & 0.770 & 0.941 & & \\
\hline Q16 & 0.545 & 0.472 & 0.700 & 0.466 & 0.589 & 0.665 & 0.694 & 0.442 & 0.529 & 0.525 & 0.806 & 0.749 & 0.820 & 0.820 & \\
\hline 217 & 0.660 & 0.631 & 0.729 & 0.631 & 0.719 & 0.744 & 0.718 & 0.647 & 0.741 & 0.453 & 0.697 & 0.680 & 0.828 & 0.831 & 0.622 \\
\hline
\end{tabular}

Elaboración propia (2017)

Es posible observar que todos los valores en la matriz de correlaciones son superiores a 0.30 por lo que el supuesto necesario para realizar el AFE se cumple en su totalidad. Adicionalmente el valor obtenido en este segundo piloto para el coeficiente alfa de Cronbach fue de 0.9611 , lo que indica nuevamente una alta consistencia y validez interna. Para realizar la validez de constructo por medio de un análisis factorial, de acuerdo a Martínez y Sepúlveda (2012) el número de factores que deben utilizarse para el análisis puede determinarse siguiendo varios criterios, el más común es el del valor propio, que representa el total de varianza explicada por el factor, para ello se utilizó el método de análisis de componentes principales y se identificaron aquellos eigenvalores mayores a 1.

La varianza poblacional es igual a la suma de los eigenvalores (valores propios), por lo que un criterio para elegir el número de componentes principales a extraer es elegir todos los componentes para los que el correspondiente valor propio (eigenvalor) sea al menos de 1, lo que implica que los componentes que permanecen representan al menos una fracción de 1/p del total de la varianza poblacional. La Figura 7 muestra el resultado. 
Figura 7. Análisis de criterio del valor propio (eigenvalor) del segundo piloto arrojado por el programa Minitab

Principal Component Analysis: Q2, Q3, Q4, Q5, Q6, Q7, Q8, Q9, Q10, Q11, Q12, Q13, Q14, Q15, Q16, Q17

Eigenanalysis of the Covariance Matrix

En Martínez y Sepúlveda (2012) se sugiere utilizar más de un criterio, por lo que adicionalmente y como método confirmatorio se utilizó el criterio de test de pendiente (scree plot), que se basa también en los valores propios, aquí se grafican las variables y se seleccionan aquellas que se encuentran antes del punto de inflexión, es decir, aquellas con valores mayores a 1. La Figura 8 muestra las gráficas correspondientes a este método.

Figura 8. Análisis del criterio del test de pendiente (scree plot) del segundo piloto arrojado por el programa Minitab

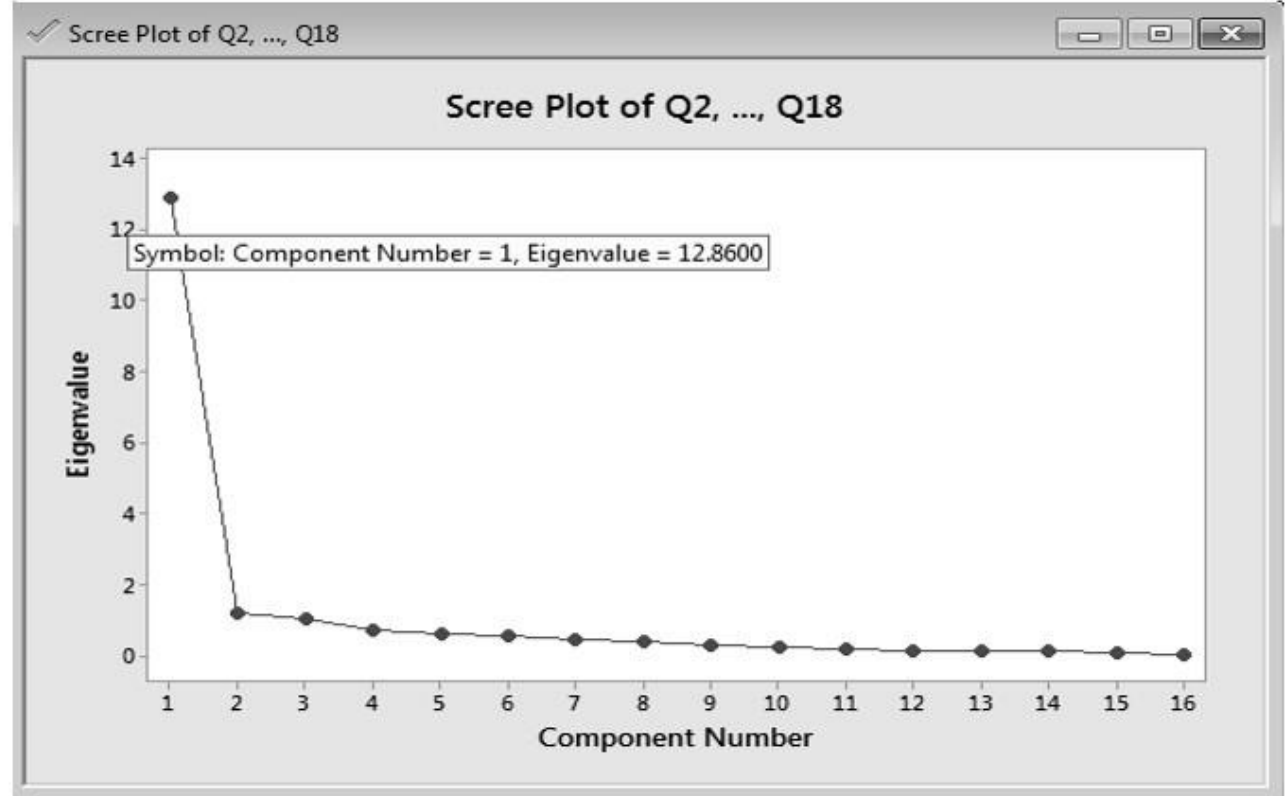

Elaboración propia (2017) 
Con los resultados de criterio del valor propio y la confirmación del criterio del test de pendiente se procedió a realizar la interpretación de los factores, por medio de la matriz correspondiente, para ello, y derivado de los análisis anteriores, se utilizó un número de tres factores. De acuerdo a Martínez y Sepúlveda (2012) en esta matriz se muestra la correlación que cada variable guarda con cada uno de los factores, un valor alto puede ser interpretado como una mayor contribución de esa variable al factor, mientras que un valor bajo indica poca contribución. Para el caso de este análisis se utilizó la rotación ortogonal varimax, que pretende maximizar las ponderaciones a nivel de factor, lo que implica que cada variable sea representativa en uno solo de ellos, de tal forma que se minimiza el número de variables que los componen. La Figura 9 muestra los resultados del AFE arrojados por el programa Minitab 17 para el segundo piloto.

\section{Figura 9. Análisis factorial exploratorio arrojado por el programa Minitab}

\begin{tabular}{|c|c|c|c|c|c|}
\hline Variable & Factorl & Factor2 & Factor 3 & Factor 4 & Communality \\
\hline Q2 & 0.252 & 0.403 & 0.629 & -0.214 & 0.667 \\
\hline Q3 & 0.176 & 0.266 & 0.842 & -0.001 & 0.810 \\
\hline Q4 & 0.474 & 0.547 & 0.380 & -0.226 & 0.719 \\
\hline Q5 & 0.281 & 0.045 & 0.787 & -0.234 & 0.755 \\
\hline Q6 & 0.504 & 0.661 & 0.202 & -0.153 & 0.755 \\
\hline Q7 & 0.542 & 0.381 & 0.312 & -0.466 & 0.753 \\
\hline Q8 & 0.664 & 0.286 & 0.174 & -0.544 & 0.848 \\
\hline Q9 & 0.181 & 0.679 & 0.310 & -0.439 & 0.783 \\
\hline Q10 & 0.354 & 0.815 & 0.187 & -0.219 & 0.872 \\
\hline Q11 & 0.266 & 0.258 & 0.152 & -0.869 & 0.915 \\
\hline Q12 & 0.845 & 0.293 & 0.160 & -0.273 & 0.899 \\
\hline Q13 & 0.779 & 0.417 & 0.188 & -0.040 & 0.817 \\
\hline Q14 & 0.746 & 0.368 & 0.387 & -0.284 & 0.922 \\
\hline Q15 & 0.740 & 0.329 & 0.437 & -0.235 & 0.901 \\
\hline Q16 & 0.824 & 0.142 & 0.308 & -0.213 & 0.838 \\
\hline Q17 & 0.511 & 0.529 & 0.526 & -0.148 & 0.839 \\
\hline Variance & 4.9762 & 3.1943 & 2.9612 & 1.9628 & 13.0945 \\
\hline of Var & 0.311 & 0.200 & 0.185 & 0.123 & 0.818 \\
\hline
\end{tabular}

Elaboración propia (2017)

Una vez que se rotaron los factores y se obtuvo la matriz de correlaciones correspondiente se procedió a evaluar las ponderaciones obtenidas en cada una de las variables para establecer la contribución de cada una de ellas a los factores. Según Martínez y Sepúlveda (2012) para realizar esta evaluación desde un punto de vista estadístico se analiza la significancia de las ponderaciones, en donde los valores 
menores a 0.3 son considerados como no significativos y sin representatividad para el factor, los valores entre 0.3 y 0.5 son considerados de representatividad mínima, los valores entre 0.5 y 0.7 se consideran de aporte significativo y finalmente los valores mayores a 0.7 son relevantes y, generalmente, son el objetivo de los análisis; sin embargo, en la práctica incluso valores por encima de 0.3 son considerados como válidos. Cabe resaltar que en todos los casos se habla tanto de valores positivos como negativos ya que la correlación entre la variable y el factor puede darse en ambos sentidos. La Tabla 2 muestra las correlaciones identificadas como resultado del análisis factorial exploratorio del segundo piloto.

Tabla 2. Resultado del análisis factorial exploratorio del segundo piloto

\begin{tabular}{ll}
\hline \multicolumn{1}{c}{ Factor } & \multicolumn{1}{c}{ Pregunta } \\
\hline Factor1 & $\mathrm{Q} 4, \mathrm{Q} 7, \mathrm{Q} 8, \mathrm{Q12}, \mathrm{Q13}, \mathrm{Q14}, \mathrm{Q15}, \mathrm{Q16}, \mathrm{Q17}$ \\
Factor 2 & $\mathrm{Q} 6, \mathrm{Q}, \mathrm{Q} 10, \mathrm{Q11}$ \\
Factor 3 & $\mathrm{Q} 2, \mathrm{Q} 3, \mathrm{Q} 5$
\end{tabular}

Fuente: Elaboración propia (2017)

\section{CONCLUSIONES}

En resumen, y de acuerdo a Mavrou (2015), al aplicar el AFE el investigador debe considerar de forma específica las siguientes cuestiones: 1) que los valores de las comunalidades sean altos para proceder, lo que se cumple cabalmente en todos los casos para el presente análisis; 2) que al determinar los factores estos incluyan idealmente al menos tres o cuatro factores, aunque en la práctica incluso dos variables podrían ser suficientes para la identificación e interpretación de un factor determinado, esta condición también se cumple para el instrumento; 3) que la magnitud de las cargas factoriales maneje saturaciones por encima del .40 .5 para considerarse satisfactorias, todas las variables manejan valores por encima de 4 en el análisis realizado; entre otras.

En base a los resultados obtenidos en el análisis factorial exploratorio se definieron los siguientes factores:

1) Beneficios del Modelo de Aprendizaje Invertido (factor 1): las preguntas que se relacionan con este factor evaluaron la utilidad percibida por los alumnos al implementar este modelo en su proceso de aprendizaje, así como la utilidad percibida 
en su desarrollo profesional, además del agrado hacia el modelo, el incremento en la motivación para realizar las actividades del curso tanto dentro como fuera el aula bajo este esquema, así como el impacto en su confianza para resolver problemas de forma autónoma bajo este modelo.

2) Uso de videos (factor 2): las preguntas que contribuyen a este factor evalúan la percepción de los estudiantes respecto a la importancia que el uso de los videos tiene en el logro de los objetivos de aprendizaje bajo el Modelo de Aprendizaje Invertido.

3) Colaboración y comunicación (factor 3): las preguntas que reflejaron una alta representatividad en este factor se relacionan con aspectos de incremento en la colaboración y comunicación tanto con los compañeros de clase como con el profesor bajo el Modelo de Aprendizaje Invertido. Es interesante analizar el hecho de que, aunque tanto la comunicación y la colaboración pueden ser consideradas como beneficios que se derivan de la implementación de este modelo, en el caso del análisis factorial las ponderaciones arrojadas por los ítems correspondientes a estos conceptos se relacionaron de tal forma que fue posible identificar un factor separado del resto de los beneficios.

Al finalizar este proceso se logró contar con un instrumento para evaluar la satisfacción y percepción de los alumnos con respecto al Modelo de Aprendizaje Invertido, aplicado de forma particular en un curso de Introducción a la Computación. Este tipo de instrumentos permiten aportar herramientas para dar sustento a los diversos beneficios que la implementación del Aprendizaje Invertido integra a las aulas, logrando con ello que cada vez más docentes de todos los niveles educativos se interesen por este tipo de innovaciones.

\section{REFERENCIAS}

Anderson, L.W., Krathwohl, D. R., Airasian, P.W., Cruikshank, K.A., Mayer, R. E., Pintrich, P. R... Wittrock, M. C. (2014). A Taxonomy for Learning, Teaching, and Assessing: Pearson New International Edition: A Revision of Bloom's Taxonomy of Educational Objectives. Harlow, Inglaterra: Pearson New International.

Bailey, A. (2015). Flipped learning. Training Journal, 5-7. Recuperado de https://www.trainingjournal.com/articles/magazine/september-2015

Bergmann, J. (2012). Myth: Flipped Learning is All About the Videos. Recuperado de http://flippedlearning.com/?p=1064

Bergmann, J., y Sams, A. (2014). Flipped-Learning Toolkit: Getting Everybody On Board [Video]. Recuperado de http://www.edutopia.org/blog/flipped-learning-getting-everybody-on-board-jonbergmann 
Bishop, J., y Verleger, M. (2013). The flipped classroom: A survey of the research. Presentado en ASEE National Conference Proceedings, Atlanta, GA.

De Araujo, Z., Otten, S., y Birisci, S. (2017). Conceptualizing "homework" in flipped mathematics classes. Journal of Educational Technology \& Society, 20(1), 248-260.

De Leeuw, E., Dillman, D., y Hox, J. (2011). International handbook of survey methodology (1st ed.). New York, NY: Psychology Press.

D'Souza, M., y Rodrigues, P. (2015). Engaging Millennial Students in an Engineering Classroom using Extreme Pedagogy. Indian Journal Of Science And Technology, 8(24). https://doi.org/10.17485/ijst/2015/v8i24/79959

Fautch, J. (2015). The flipped classroom for teaching organic chemistry in small classes: is it effective?. Chemistry Education Research and Practice, 16(1), 179-186.

Flipped Learning Network. (2014). The Four Pillars of F-L-I-P. Recuperado de http://www.flippedlearning.org/cms/lib07/VA01923112/Centricity/Domain/46/FLIP_handout_FNL_ Web.pdf

Flumerfelt, S., y Green, G. (2013). Using Lean in the Flipped Classroom for At Risk Students. Journal Of Educational Technology \& Society, 16(1), 356-366.

Franklin, S., y Walker, C. (2010). Survey methods and practices (1st ed.). Ottawa, Canadá: Statistics Canada.

González-Gómez, D., Jeong, J., Airado, D., y Cañada-Cañada, F. (2016). Performance and perception in the flipped learning model: An initial approach to evaluate the effectiveness of a new teaching methodology in a general science classroom. Journal of Science Education and Technology, 25(3), 450-459. https://doi.org/10.1007/s10956-016-9605-9

Gillispie, V. (2016). Using the flipped classroom to bridge the gap to generation Y. The Ochsner Journal, 16(1), 32-36. Recuperado de https://www.ncbi.nlm.nih.gov/pubmed/27046401

Hamdan, N., McKnigth, K., y Arfstrom, K. (2014). Extension of a review of flipped learning. Recuperado de http://flippedlearning.org/wp-content/uploads/2016/07/Extension-of-FLipped-Learning-LIt-ReviewJune-2014.pdf

Hao, Y. (2016). Exploring undergraduates' perspectives and flipped learning readiness in their flipped classrooms. Computers in Human Behavior, 59, 82-92. https://doi.org/10.1016/j.chb.2016.01.032

Hernández, R., Fernández, C., y Baptista, P. (2014). Metodología de la investigación. México, D.F., México: McGraw-Hill Education.

Herreid, C., y Schiller, N. (2013). Case studies and the flipped classroom. Journal of College Science Teaching, 42(5), 62-66.

Joanne, C., y Lateef, F. (2014). The Flipped Classroom: Viewpoints in Asian Universities. Education In Medicine Journal, 6(4), e20-e26. https://doi.org/10.5959/eimj.v6i4.316

Johnson, L., Adams, S., Estrada, V., y Freeman, A. (2014). NMC Horizon Report: 2014 Higher Education Edition. Austin, TX: The New Media Consortium. 
Johnson, L., Adams, S., Estrada, V., y Freeman, A. (2015). NMC Horizon Report: 2015 Higher Education Edition. Austin, TX: The New Media Consortium.

Martínez, C., y Sepúlveda, M. (2012). Introducción al análisis factorial exploratorio. Revista Colombiana De Psiquiatría, 41(1), 197-207. https://doi.org/10.1016/s0034-7450(14)60077-9

Mavrou, I. (2015). Análisis factorial exploratorio: cuestiones conceptuales y metodológicas. Revista Nebrija de Lingüística Aplicada, 9(4). Recuperado de https://www.nebrija.com/revistalinguistica/files/articulosPDF/articulo_56583a3b9a601.pdf

McLean, S., Attardi, S., Faden, L., y Goldszmidt, M. (2016). Flipped classrooms and student learning: Not just surface gains. Advances in Physiology Education, 40(1), 47-55.

McMahon, M., y Pospisil, R. (2005). Laptops for a digital lifestyle: millennial students and wireless mobile technologies. Recuperado de http://citeseerx.ist.psu.edu/viewdoc/download?doi=10.1.1.504.9796\&rep=rep1\&type=pdf

Murphy, J., Chang, J., y Suaray, K. (2016). Student performance and attitudes in a collaborative and flipped linear algebra course. International Journal of Mathematical Education in Science and Technology, 47(5), 653-673. https://doi.org/10.1080/0020739X.2015.1102979

Ojennus, D. (2016). Assessment of learning gains in a flipped biochemistry classroom. Biochemistry and Molecular Biology Education, 44(1), 20-27. https://doi.org/10.1002/bmb.20926

Oviedo, H. C., y Campo, A. (2005). Aproximación al uso del coeficiente alfa de Cronbach. Revista Colombiana de Psiquiatría, 34(4), 572-580.

Ozdamli, F., y Asiksoy, G. (2016). Flipped classroom approach. World Journal on Educational Technology, 8(2), 98-105. https://doi.org/10.18844/wjet.v8i2.640

Pérez-Gil, J., Chacón, S., y Moreno, R. (2000). Validez de constructo: El uso del análisis factorial exploratorio-confirmatorio para obtener evidencias de validez. Psicothema, 12(2), 442-446.

Prensky, M. (2001). Digital Natives, Digital Immigrants Part 1. On The Horizon, 9(5), 1-6. https://doi.org/10.1108/10748120110424816

Sams, A., y Aglio, J. (2017). Three ways the flipped classroom leads to better subject mastery. The Education Digest, 82(5), 52-54.

Sarawagi, N. (2013). Flipping an introductory programming course: yes you can!. Journal of Computing Sciences in Colleges, 28(6),186-188.

Thai, N., De Wever, B., y Valcke, M. (2017). The impact of a flipped classroom design on learning performance in higher education: Looking for the best "blend" of lectures and guiding questions $\begin{array}{llll}\text { with feedback. Computers \& } \quad \text { Education, } & 107,126 .\end{array}$ https://doi.org/10.1016/j.compedu.2017.01.003

Tucker, B. (2012). The flipped classroom. Education Next, 12(1), 82-83. 